\title{
SEISMIC DAMAGE ANALYSIS OF REINFORCED CONCRETE BUILDING CONSIDERING MEMBER FRACTURE
}

\author{
Daigoro ISOBE \\ (Assistant Professor, Institute of Engineering Mechanics, University of Tsukuba, \\ 1-1-1 Tennodai Tsukuba-shi, Ibaraki 305 Japan)
}

\begin{abstract}
The Adaptively Shifted Integration (ASI) technique, which produces the highest computational efficiency in the finite element analyses of framed structures including static and dynamic collapse problems, is applied to the seismic damage analysis of a reinforced concrete building. By expressing member fracture by a plastic hinge located at the exact position with a simultaneous release of resultant forces in the element, discontinuous problem such as this kind can be easily analyzed even by the conventional finite element code with the displacemental form. By using the algorithms described in this paper, sufficiently reliable solutions for the practical use have been obtained in the seismic damage analysis of a five stories-five span reinforced concrete building. The present technique can be easily implemented with a minimum effort into the existing finite element codes utilizing the linear Timoshenko beam element.
\end{abstract}

\section{INTRODUCTION}

The Great Hanshin-Awaji Earthquake, which occurred in January 1995, had given major damage over the area. Thus the structural design guidelines for buildings especially against vertical seismic wave has been thoroughly reconsidered. Therefore, the arrival of a convenient technique to analyze collapse modes of structural objects under three-directional excitation is now desired.

However, there are few numerical examples applying computational schemes such as the Distinct Element Method (DEM)[1][3] or the Discontinuous Deformation Analysis (DDA)[2][4] to seismic damage analyses. On the other hand, generally used finite element code needs some extra complicated processes to simulate this kind of dynamic collapse problem, which contains strong nonlinearity and discontinuity such as member fracture, occurred in flexural damage or shear damage in reinforced concrete members.

In this paper, the Adaptively Shifted Integration (ASI) technique with the linear Timoshenko beam element, which can be easily implemented with a minimum effort into the existing finite element codes, is applied to the seismic damage analysis of a reinforced concrete building. As shown in the previous papers[6][7], the highest computational efficiency in the finite element analyses of framed structures including the geometrically nonlinear, elasto-plastic analysis as well as the geometrically linear, plastic collapse analysis and the dynamic collapse analyses have been produced by using the ASI technique.

In this technique, the numerical integration point in an elastically deformed element is placed at the optimal point for linear analysis(midpoint in the linear Timoshenko beam), while the integration point is shifted immediately after the occurrence of a fully-plastic section in the element, using the previously established relation between the location of numerical integration point and that of plastic hinge, to form a plastic hinge exactly at the position of that section. Thus the technique produces higher computational accuracy with fewer elements than the conventional finite element code. Member fracture can be expressed by a plastic hinge located at the exact position with a simultaneous release of resultant forces in the element. The expression of a fracture at one side of member section is possible by utilizing at least two Timoshenko beam elements as a member. With the proposed technique, discontinuous problems can be 
easily analyzed even by the conventional finite element code with the displacemental form.

\section{ASI TECHNIQUE FOR DYNAMIC COLLAPSE ANALYSIS}

In this chapter, the algorithm of the ASI technique, which is applied to the dynamic collapse analysis, is explained. By considering the equivalence conditions between the strain energy approximations of the linear Timoshenko beam element and the physical model, the rigid-bodies spring model (RBSM), the relation between the location of a numerical integration point $\left(\mathrm{s}_{1}\right)$ and that of occurrence of a plastic hinge $\left(\mathrm{r}_{1}\right)$ in the linear Timoshenko beam element $\left(-1 \leq r_{1}, s_{1} \leq 1\right)$ is obtained. Referring to Fig. 1 , it is expressed by the following equation:

$$
s_{1}=-r_{1} \text { or } r_{1}=-s_{1}
$$

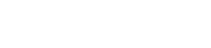

where $s_{1}$ and $r_{1}$ are the position of the numerical integration point and the position of the plastic hinge or the fracture to be formed, respectively.

When the entire region in an element behaves elastically, the midpoint of the element $\left(s_{1}=0\right)$ is the most adequate integration point from the point of view of accuracy and symmetry. Thus the internal force vector at incremental step $n$ based on the Updated Lagrangian Formulation (U.L.F.) is expressed as

$$
\left\{{ }_{n}^{n} F\right\}=\int_{{ }^{n} l}\left[{ }^{0} T\right]^{T} \cdot\left[{ }^{u} T\right]^{T} \cdot\left[{ }_{n}^{n} \overline{B_{L}}(0)\right]^{T} \cdot\left\{\begin{array}{l}
n \\
n
\end{array} \bar{R}(0)\right\} d l
$$

where the value in a parenthesis in $\left[{ }_{n}^{n} \bar{B}_{L}\right]$ indicates the location of the integration point, and that in $\{n \bar{R}\}$ indicates the point at which stresses are evaluated, respectively. According to the elementary beam theory, the relations between bending moments $\bar{R}_{1}$, $\bar{R}_{2}$ and shear forces $\bar{R}_{5}, \bar{R}_{6}$ are expressed as

$$
\bar{R}_{5}=-\frac{d \bar{R}_{2}}{d z}, \quad \bar{R}_{6}=-\frac{d \bar{R}_{1}}{d z}
$$

Thus the distributions of the bending moment increments $\Delta_{n} \bar{R}_{1}(s)$ and $\Delta_{n} \bar{R}_{2}(s)$ along the element length can be approximated by the following equations using the bending moment increments $\Delta_{n} \bar{R}_{1}(0), \Delta_{n} \bar{R}_{2}(0)$ and the shear force increments $\Delta_{n} \bar{R}_{5}(0)$, $\Delta_{n} \bar{R}_{6}(0)$ at the midpoint in the element:

$$
\Delta_{n} \bar{R}_{1}(s)=\Delta_{n} \bar{R}_{1}(0)-\frac{\Delta_{n} \bar{R}_{6}(0)^{n} l s}{2}, \quad \Delta_{n} \bar{R}_{2}(s)=\Delta_{n} \bar{R}_{2}(0)-\frac{\Delta_{n} \bar{R}_{5}(0)^{n} l s}{2}
$$

where ${ }^{n} l$ is the element length at $t=t_{n}$. Equations (2.4) show that the bending moments are subject to a linear change in an element and are likely to take the maximum value on either ends $(s= \pm 1)$. As other resultant forces have constant values in the element, a fully-plastic state can be determined with a yield function by comparing the calculated distributions from equations (2.4).

In the dynamic collapse analyses using the ASI technique, member fracture is expressed by shifting the numerical integration point according to equation (2.1) immediately after the occurrence of a fractured section on either end of the element, and by reducing the resultant forces of the element simultaneously. For instance, if the fully-plastic section or the fractured section has first occurred at the left edge in the element $(\mathrm{r}=-1)$, the numerical integration point is shifted immediately to the right edge in the element $(s=1)$ according to equation (2.1). The released force vector, which operates to the element at the following incremental step is then expressed by the following equation: 


$$
\left\{{ }_{n}^{n} F\right\}=\int_{{ }_{n} l}\left[{ }^{0} T\right]^{T} \cdot\left[{ }^{u} T\right]^{T} \cdot\left[{ }_{n}^{n} \overline{B_{L}}(1)\right]^{T} \cdot\left\{{ }_{n}^{n} \bar{R}(-1)\right\} d l
$$

Similarly, if the fully-plastic section or the fractured section has first occurred at the right edge in the element $(r=1)$, the numerical integration point is shifted to the left edge in the element $(s=-1)$.

In case of the implicit scheme, the following incremental stiffness matrices and initial stress matrices are used in the algorithm:

$$
\begin{aligned}
& \left.\left.\left[{ }_{n}^{n} \bar{K}_{L}\right]=\int_{{ }_{n} l}\left[{ }^{u} T\right]^{T} \cdot\left[{ }^{0} T\right]^{T}\left[{ }_{n}^{n} \overline{B_{L}}(0)\right]^{T}\left[D^{e}(0)\right]\right]_{n}^{n} \overline{B_{L}}(0)\right]\left[\left[^{0} T\right] \cdot\left[{ }^{u} T\right] d l\right. \\
& {\left[{ }_{n}^{n} \bar{K}_{N L}\right]=\int_{{ }_{n} l}\left[{ }^{u} T\right]^{T} \cdot\left[{ }^{0} T\right]^{T}\left[{ }^{n} \bar{G}(0)\right]^{T}\left[{ }^{n} \bar{S}(0)\right]\left[{ }^{n} \bar{G}(0)\right]\left[{ }^{0} T\right] \cdot\left[{ }^{u} T\right] d l}
\end{aligned}
$$

if the entire region in an element behaves elastically, and

$$
\begin{aligned}
& {\left[{ }_{n}^{n} \bar{K}_{L}\right]=\int_{{ }_{n} l}\left[{ }^{u} T\right]^{T} \cdot\left[{ }^{0} T\right]^{T}\left[{ }_{n}^{n} \overline{B_{L}}(1)\right]^{T}\left[D^{e}(-1)\right]\left[_{n}^{n} \overline{B_{L}}(1)\right]\left[\left[^{0} T\right] \cdot\left[{ }^{u} T\right] d l\right.} \\
& {\left[{ }_{n}^{n} \bar{K}_{N L}\right]=\int_{{ }_{n} l}\left[{ }^{u} T\right]^{T} \cdot\left[{ }^{0} T\right]^{T}\left[{ }_{n}^{n} \bar{G}(1)\right]^{T}\left[{ }^{n} \bar{S}(-1)\right]\left[{ }_{n}^{n} \bar{G}(1)\right]\left[{ }^{0} T\right] \cdot\left[{ }^{u} T\right] d l}
\end{aligned}
$$

if the fully-plastic section or the fractured section has first occurred at the left edge in the element $(\mathrm{r}=-1)$.

At the fracture phase in an element, the numerical integration point is shifted to the opposite end of the fractured section from the center of the element. Therefore, an imaginary plastic hinge occurs at the fractured section according to the algorithm explained in this chapter. Generally, to release the resultant forces instantly is considered not the best way as the longitudinal wave might occur in the process. In such cases, a gradual release technique or a technique considering artificial viscosity is used to stabilize the numerical calculation. However, in this paper, an instant release technique is used to make the algorithm simple. Rebounds and insertions to the ground, or contacts between elements are neglected in this algorithm.

It is also to be noted that as elements and node points are still treated as a continuous model in the calculation process, new imaginary node points for the fractured sections are needed to be established at the post processing stage. The elements with the imaginary node points are then visualized as rigid bars thereafter.

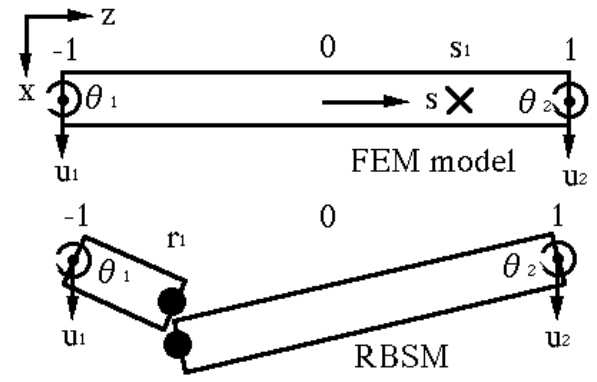

$\times$ Numerical integration point

- Rotational and shear spring connecting rigid bars (plastic hinge)

Fig.1 Linear Timoshenko beam element and its physical equivalent

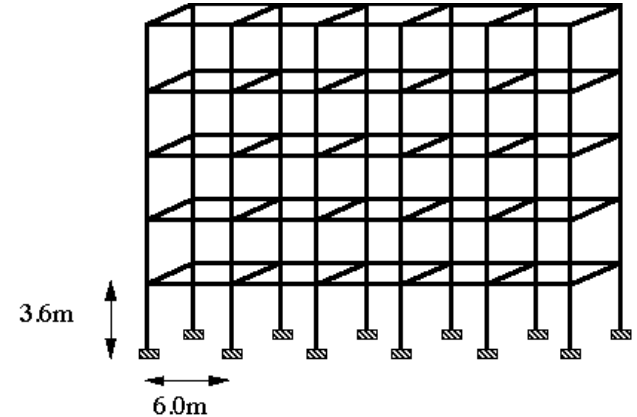

Fig.2 Reinforced concrete building model used in the seismic damage analysis 


\section{SEISMIC DAMAGE ANALYSIS OF A REINFORCED CONCRETE BUILDING}

In this chapter, the implicit nonlinear code based on the U.L.F. with the ASI technique is implemented into the existing finite element code, and the code has been applied to the seismic damage analysis of a five stories-five span reinforced concrete building as shown in Fig.2.

Generally, many parameters such as shape, steel bar arrangement, material characteristics as well as stress condition or stress history, contribute big influence to the strength characteristics of a reinforced concrete member. Consequently, the limits of application range occur with nature for proposal formulas and experimental data. In this paper, the formulas, which are partially excluded for these restrictive matters, are used [8].

Dead loads are given statically to the node points at initial step of the analysis. The process is followed by dynamic analysis under excitation at fixed points by threedirectional seismic wave (Great Hanshin-Awaji Earthquake, 1995, JMA Kobe), as shown in Fig.3. A summary of the analyzed model is as follows; Young's modulus of concrete $E_{c}=2.1 \times 10^{5} \mathrm{~kg} / \mathrm{cm}^{2}$, Poisson's ratio of concrete $v_{c}=0.17$, density of reinforced concrete member $\rho=2.4 \times 10^{-2} \mathrm{~kg} / \mathrm{cm}^{3}$, compressive strength of concrete $F_{c}=240 \mathrm{~kg} / \mathrm{cm}^{2}$, yield stress of tension reinforcement ${ }_{s} f_{y}=4.0 \times 10^{3} \mathrm{~kg} / \mathrm{cm}^{2}$, tension yield stress of shear reinforcement ${ }_{s} f_{w y}=3.0 \times 10^{3} \mathrm{~kg} / \mathrm{cm}^{2}$. Fracture of the members is assumed to occur at the critical curvature point $\left(\kappa_{c r}=1.0 \times 10^{-3} 1 / \mathrm{mm}\right)$. The column section of $1 \mathrm{st}$ floor is $80 \mathrm{~cm} \times 80 \mathrm{~cm}$, and that of over 2 nd floor is $60 \mathrm{~cm} \times 60 \mathrm{~cm}$, both squarely shaped. A $40 \mathrm{~cm} \times 40 \mathrm{~cm}$ reinforced concrete member is used for every lateral beam. Main reinforcement ratio of the columns is $4 \%$ for below 3rd floor, $0.8 \%$ (minimum allowable ratio) for upper floors, and $5 \%$ for lateral beams, respectively. The diameter of shear reinforcement in every member is set to $1.3 \mathrm{~cm}$, and the distance between each shear reinforcements $\chi$ is given as $30 \mathrm{~cm}$.

Newmark's $\beta$ method $(\delta=1 / 2)$, well known for its practicability and usefulness, is used for the direct numerical integration scheme in this analysis. However, results tend to emit in range of $0<\beta \leq 1 / 4$ for sake of its strong nonlinearity of the problem. Therefore $\beta=0.4$, which is in range of $1 / 4<\beta<1 / 2$ where solutions are likely to be stable though precision falls a little [9], is used in the analysis. Time increment used in

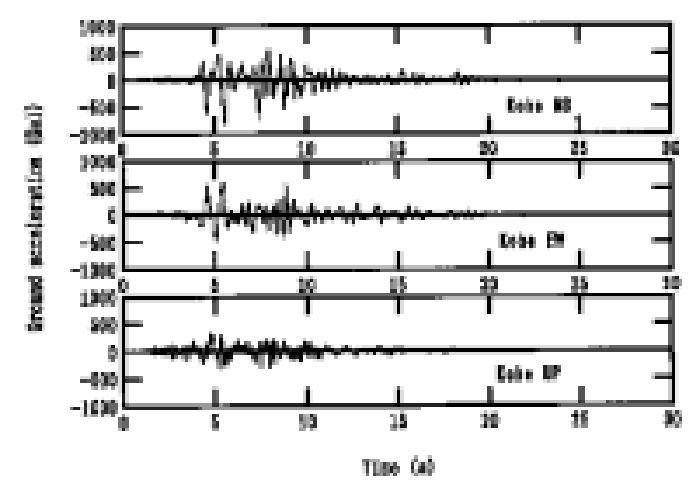

Fig. 3 Great Hanshin-Awaji Earthquake accelerogram voed in the analysis

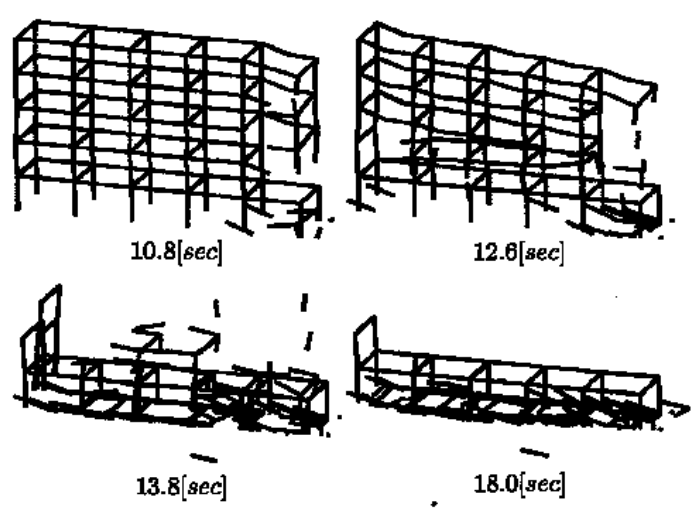

Fig.4 Seismic damage analysis of reinforced concrete building using ASI technique 
this analysis is $\Delta t=7.50 \times 10^{-3}[\mathrm{sec}]$, and input time of the seismic wave is $30[$ sec $](4000$ steps). The computing time on the EWS (Sun SPARC station 5) is about 190 minutes.

Figure 4 shows the collapse process of a reinforced concrete building under the Great Hanshin-Awaji seismic wave. Member fracture occurs first at a column member on second floor, which has lost their strength by repetition of yielding and unloading, followed by a collapse chain reaction of columns and beams supporting third floor and above. Appearance of the mode collapsing from upper floor is inevitable because the main reinforcement ratio of the columns upper floor is reduced to the minimum allowable ratio, which is actually a commonly used value in Japan. The mode which differ from actual phenomenon such as beams going through a floor appears in this analysis, which is due to the disregard of contacts between elements.

As a result of the analysis, it is estimated that the expression of the seismic damage process of reinforced concrete building has become possible by using the algorithm shown in this paper. On the other hand, the algorithm is confirmed to be practical and economic by using the Conjugate Gradient method as solver, though the implicit nonlinear code is used.

\section{CONCLUDING REMARKS}

In the present paper, the nonlinear finite element code using the ASI technique, which can express a plastic hinge or a fractured section exactly at the end of an element, is applied to the seismic damage analysis of a reinforced concrete building.

The case of five stories-five span reinforced concrete building under excitation by the accelerogram of Great Hanshin-Awaji Earthquake is studied. The practical expression of the damage process has been obtained in the analysis. It is confirmed that the algorithm can be applied to the structural design process of buildings.

The strong nonlinear problems, in which complicated processes are needed in the conventional finite element code to analyze, can be easily analyzed by implementing the ASI technique to the finite element codes utilizing the linear Timoshenko beam element.

This research is partially in support of the University of Tsukuba Research Projects.

\section{REFERENCES}

[1] P.A.Cundall, A Computer Model for Simulating Progressive, Large-scale Movement in Blocky Rock System, Proceedings of the International Symposium on Rock Mechanics, II-8,129-136,(1971).

[2] G.H.Shi and R.E.Goodman, Discontinuous Deformation Analysis, Proceedings of 25th U.S. Symposium on Rock Mechanics, 269-277,(1984).

[3] K.Meguro and M.Hakuno, Simulation of Collapse Process of Structures due to Earthquake, Proceedings of Symposium on Computational Methods in Structural Engineering and Related Fields, Vol.15,325-330,(1991).[in Japanese]

[4] M.Y.Ma, P.Barbeau and D.Penumadu, Evaluation of active thrust on retaining walls using DDA, Journal of Computing in Civil Engineering, Vol.1,820-827,(1995).

[5] Y.Toi, Shifted Integration Technique in one-dimensional plastic collapse analysis using linear and cubic finite elements, International Journal for Numerical Methods in Engineering,Vol.31,1537-1552,(1991).

[6] Y.Toi and D.Isobe, Adaptively Shifted Integration Technique for Finite Element Collapse Analysis of Framed Structures, International Journal for Numerical Methods in Engineering,Vol.36,2323-2339,(1993).

[7]Y.Toi and D.Isobe, Finite Element Analysis of Quasi-static and Dynamic Collapse Behaviors of Framed Structures by the Adaptively Shifted Integration Technique, Computers and Structures, Vol.58,947-955,(1996).

[8] Architectural Institute of Japan, Guidelines on ultimate strength design of reinforced concrete,70-71.[in Japanese]

[9] N.Shimizu, Response analysis using personal computers,Kyouritu-Shuppan,267-268.[in Japanese] 